\title{
Frequency-Domain Multiuser Detection for Highly Overloaded DS-CDMA Systems
}

\author{
Paulo Silva \\ IT/ISE, Universidade do Algarve \\ Campus da Penha, Faro, Portugal \\ Email: psilva@ualg.pt
}

\author{
Rui Dinis \\ IT/FCT, Universidade Nova de Lisboa \\ Monte da Caparica, Portugal \\ Email: rdinis@fct.unl.pt
}

\begin{abstract}
A DS-CDMA (Direct Sequence-Coded Division Multiple Access) system has maximum spectral efficiency if the system is fully loaded (i.e., the number of users is equal to the spreading factor) and we employ signals with bandwidth equal to the chip rate. However, due to implementation constraints we need to employ signals with higher bandwidth, decreasing the system's spectral efficiency. In this paper we consider prefixassisted DS-CDMA systems with bandwidth that can be significantly above the chip rate. To allow high spectral efficiency we consider highly overloaded systems where the number of users can be twice the spreading factor or even more. To cope with the strong interference levels we present an iterative frequencydomain receiver that takes full advantage of the total bandwidth of the transmitted signals. Our performance results show that the proposed receiver can have excellent performance, even for highly overloaded systems. Moreover, the overall system performance can be close to the maximum theoretical spectral efficiency, even with transmitted signals that have bandwidth significantly above the chip rate. ${ }^{1}$
\end{abstract}

\section{INTRODUCTION}

Prefix-assisted DS-CDMA schemes (Direct SequenceCoded Division Multiple Access) are suitable for broadband wireless systems since they combine the flexibility and capacity of CDMA schemes with the possibility of efficient frequency-domain receiver implementations, making them appropriate for severely time-dispersive channels [1]. Since the transmitted signals have single-carrier nature, they can be designed to have reduced envelope fluctuations, making them particularly interesting for the uplink transmission [i.e., the transmission from the mobile terminals to the base stations (BS)] [2], [3]. However, the receiver design presents some challenges at the uplink transmission since a different channel affects the signal associated to each transmitter. This means that the interference levels can be severe and some kind of multiuser detection is required. A promising detection scheme for DS-CDMA was proposed in [4] which can be regarded as an iterative frequency-domain multiuser detection scheme based on the IB-DFE (Iterative Block-Decision Feedback Equalizer) concept [5], allowing performance close to the MFB (Matched Filter Bound). It is well known that a DSCDMA system has maximum spectral efficiency if the system is fully loaded (i.e., the number of users is equal to the spreading factor) and we employ signals with bandwidth equal to

\footnotetext{
${ }^{1}$ This work was partially supported by the FCT (Fundação para a Ciência e Tecnologia) project MPSat PTDC/EEA-TEL/099074/2008.
}

the chip rate, i.e., by employing raised-cosine pulses with zero roll-off. However, it is much simpler to implement pulses with larger bandwidth (e.g., square-root raised-cosine pulses with roll-off one or pulses with chip duration), the synchronization is simpler and the signals are more robust to synchronization errors. Moreover, the envelope fluctuations and the PAPR (Peak-to-Average Power Ratio) of the transmitted signals are lower for pulses with larger bandwidth. Clearly, the major problem inherent to adopting pulses with bandwidth above the chip rate is that we decrease the overall spectral efficiency of the system. In fact, if we duplicate the bandwidth (as when we employ square-root raised-cosine pulses with roll-off one) the spectral efficiency is reduced by a factor of 2 . However, if we duplicate the number of users (i.e., if the number of users is twice the spreading factor, corresponding to a system load of $200 \%$ ) we can maintain the overall system's spectral efficiency. However, the interference levels in highly overloaded systems preclude good performance.

In this paper we consider prefix-assisted DS-CDMA systems with bandwidth that can be significantly above the chip rate. To allow high spectral efficiency we consider highly overloaded systems where the number of users can be twice the spreading factor or even more. To cope with the strong interference levels we present an iterative frequency-domain receiver that takes full advantage of the total bandwidth of the transmitted signals. This paper is organized as follows: Sec. II describes the system model and Sec. III addresses the multiuser frequency-domain receiver for joint equalization and interference cancelation considered in this paper. Sec. IV presents a set of performance results and Sec. V is concerned with the conclusions of the paper.

\section{SySTEM MOdeL}

In this paper we consider the uplink transmission in DSCDMA systems employing cyclic prefix-assisted (CP) block transmission techniques where the transmission bandwidth is above the chip rate. We have a spreading factor $K$ and $P$ users. It is assumed that the received blocks associated to each user are synchronized in time (in practice, this means that there is a suitable "time-advance" mechanism allowing perfect synchronization, although just a coarse synchronization is required since some time misalignments can be absorbed by the $\mathrm{CP}$ ). 
The time-domain block of chips to be transmitted by the $p$ th user has the form

$$
s_{p}(t)=\sum_{n=-N_{G}}^{N-1} s_{n, p} \xi_{p} h^{(T)}\left(t-n T_{C}\right)
$$

with $T_{C}$ denoting the chip duration, $N_{G}$ denoting the number of samples at the $\mathrm{CP}, \xi_{p}$ is a suitable scale factor that accounts for the overall attenuation between the $p$ th user and the BS and $h^{(T)}(t)$ is the adopted pulse shape, which has bandwidth $J$ times above the chip rate. The size- $M$ data block to be transmitted by the $p$ th is $\left\{a_{m, p} ; m=0,1, \ldots, M-1\right\}$, where $a_{m, p}$ is the $m$ th data symbol, selected from a given constellation under an appropriate mapping rule and $a_{-m, p}=$ $a_{M-m, p}$. The corresponding chip block to be transmitted is $\left\{s_{n, p} ; n=0,1, \ldots, N-1\right\}$, where $N=M K$ and

$$
s_{n, p}=a_{\lfloor n / K\rfloor, p} c_{n, p}
$$

( $\lfloor x\rfloor$ denotes "larger integer not higher than $x$ "), with $c_{n, p}$ denoting the spreading symbols. It is assumed that the spreading sequence is periodic with period $K$ (i.e., $c_{n+K, p}=c_{n, p}$ ).

The received overall signal associated to each user is sampled $J$ times each chip (i.e., the sampling rate is $J / T_{C}$ ) and the $\mathrm{CP}$ is removed, leading to the time-domain received block $\left\{y_{n} ; n=0,1, \ldots, J N-1\right\}$. It can be shown that, when the $\mathrm{CP}$ is longer than the overall channel impulse response, the corresponding frequency-domain block is $\left\{Y_{k} ; k=\right.$ $0,1, \ldots, J N-1\}$, where

$$
Y_{k}=\sum_{p=1}^{P} S_{k, p}^{(J)} \xi_{p} H_{k, p}^{(C h)}+N_{k}
$$

with $H_{k, p}^{(C h)}$ denoting the channel frequency response for the $p$ th and the $k$ th frequency and $N_{k}$ is the channel noise for that frequency. The frequency-domain block $\left\{S_{k, p}^{(J)} ; k=\right.$ $0,1, \ldots, J N-1\}$ is the discrete Fourier transform (DFT) of the augmented chip block $\left\{s_{n, p}^{(J)} ; n=0,1, \ldots, J N-1\right\}$ generated by adding zeros to the transmitted chip block $\left\{s_{n, p} ; n=0,1, \ldots, N-1\right\}$ in the following way:

$$
s_{n, p}^{(J)}=\left\{\begin{array}{ll}
s_{n^{\prime}, p}, & n=n^{\prime} J, \\
0, & \text { otherwise }
\end{array} .\right.
$$

Therefore, the frequency-domain block $\left\{S_{k, p}^{(J)} ; k=\right.$ $0,1, \ldots, J N-1\}$ is the size- $J N$ periodic extension of the DFT of the block of chips associated to the $p$ th user $\left\{S_{k, p} ; k=0,1, \ldots, N-1\right\}$. Since $s_{n, p}=a_{\lfloor n / K\rfloor, p} c_{n, p}$, it can be easily shown that

$$
S_{k, p}=A_{k, p}^{\prime} C_{k, p}^{\prime}
$$

where $\left\{A_{k, p}^{\prime} ; k=0,1, \ldots, N-1\right\}$ is the DFT of $\left\{a_{n, p}^{\prime} ; n=\right.$ $0,1, \ldots, N-1\}$, with

$$
a_{n, p}^{\prime}= \begin{cases}a_{m, p}, & n=m K \\ 0, & \text { otherwise }\end{cases}
$$

and $\left\{C_{k, p}^{\prime} ; k=0,1, \ldots, N-1\right\}$ is the DFT of $\left\{c_{n, p}^{\prime} ; n=\right.$ $0,1, \ldots, N-1\}$, with

$$
c_{n, p}^{\prime}= \begin{cases}c_{n, p}, & 0 \leq n<K \\ 0, & \text { otherwise }\end{cases}
$$

Clearly,

$$
A_{k, p}^{\prime}=\frac{1}{K J} A_{k \bmod M, p},
$$

$k=0,1, \ldots, N-1,(x \bmod y$ denote modulus operator $)$ with $\left\{A_{k, p} ; k=0,1, \ldots, M-1\right\}$ the DFT of $\left\{a_{m, p} ; m=\right.$ $0,1, \ldots, M-1\}$. This means that, apart a constant, the block $\left\{A_{k, p}^{\prime} ; k=0,1, \ldots, N-1\right\}$ is the size- $N$ periodic extension of the DFT of the data block associated to the $p$ th user $\left\{A_{k, p} ; k=\right.$ $0,1, \ldots, M-1\}$. This multiplicity in the $A_{k, p}^{\prime}$ is related to the spectral correlations that are inherent to the cyclostationary nature of the transmitted signals [6]. Therefore,

$$
Y_{k}=\sum_{p=1}^{P} A_{k \bmod M, p} H_{k, p}+N_{k},
$$

with

$$
H_{k, p}=\frac{1}{K J} \xi_{p} H_{k, p}^{(C h)} C_{k, p}^{\prime}
$$

denoting the equivalent channel frequency response of the $p$ th user for the $k$ th frequency. In matrix notation, (9) is equivalent to

$$
\mathbf{Y}(k)=\mathbf{H}^{T}(k) \mathbf{A}(k)+\mathbf{N}(k)
$$

$\left[(\cdot)^{T}\right.$ denote the transpose matrix $]$, with

$$
\begin{aligned}
\mathbf{Y}(k) & =\left[\begin{array}{llll}
Y_{k} & Y_{k+M} & \cdots & Y_{k+(K J-1) M}
\end{array}\right]^{T}, \\
\mathbf{N}(k) & =\left[\begin{array}{llll}
N_{k} & N_{k+M} & \cdots & N_{k+(K J-1) M}
\end{array}\right]^{T}, \\
\mathbf{A}(k) & =\left[\begin{array}{llll}
A_{k} \bmod M, 1 & \cdots & A_{k \bmod M, P}
\end{array}\right]^{T}
\end{aligned}
$$

and

$$
\mathbf{H}(k)=\left[\begin{array}{c}
\mathbf{H}_{1}(k) \\
\vdots \\
\mathbf{H}_{P}(k)
\end{array}\right]=\left[\begin{array}{ccc}
H_{k, 1} & \cdots & H_{k+(K J-1) M, 1} \\
\vdots & & \vdots \\
H_{k, P} & \cdots & H_{k+(K J-1) M, P}
\end{array}\right] .
$$

Since we have $K J$ replicas associated to each $A_{k, p}$ we can separate $P=K J$ different users at the BS.

\section{RECEIVER STRUCTURE}

We consider an iterative frequency-domain multiuser receiver based on the receiver proposed in [7]. Each iteration consists of $P$ detection stages, one for each user. It is assumed that the users are ordered in descending order of their power and, when detecting a given user, the interference from the other users is canceled, as well as the residual ISI for the user that is being detected. These interference and residual ISI cancelations take into account the reliability of each of the previously detected users.

For a given iteration, the detection of the $p$ th user employs the structure depicted in Fig. 1, where we have one length$N$ feedforward filter, followed by a decimation procedure and $P$ length- $M$ feedback filters (one for each user). The 


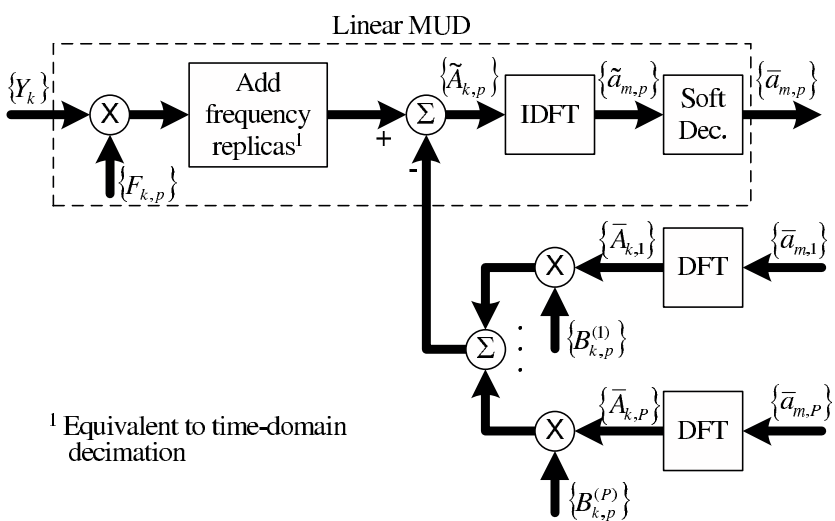

Fig. 1. Detection of the $p$ th user.

feedforward filters are designed to minimize both the ISI and the interference that cannot be canceled by the feedback filters, due to decision errors in the previous detection steps. After an inverse DFT (IDFT) operation, the corresponding timedomain outputs are passed through a soft-decision device so as to provide an estimate of the data block transmitted by the $p$ th user. For the case where we do not have any information about the users' data blocks, the receiver reduces to a linear frequency-domain MUD.

For each iteration, the frequency-domain samples associated with the $p$ th user at the detector output are given by

$$
\tilde{A}_{k, p}=\mathbf{F}_{p}^{T}(k) \mathbf{Y}(k)-\mathbf{B}_{p}^{T}(k) \overline{\mathbf{A}}(k)
$$

where the vector

$$
\begin{gathered}
\overline{\mathbf{A}}(k)=\left[\begin{array}{llll}
\bar{A}_{k \bmod M, 1} & \cdots & \bar{A}_{k \bmod M, P}
\end{array}\right]^{T}, \\
\mathbf{F}_{p}(k)=\left[\begin{array}{llll}
F_{k, p} & F_{k+M, p} & \cdots & F_{k+(K J-1) M, p}
\end{array}\right]^{T}
\end{gathered}
$$

and

$$
\mathbf{B}_{p}(k)=\left[\begin{array}{lll}
B_{k, p}^{(1)} & \cdots & B_{k, p}^{(P)}
\end{array}\right]^{T} .
$$

The block $\left\{\bar{A}_{k, p} ; k=0,1, \ldots, M-1\right\}$ is the DFT of the block $\left\{\bar{a}_{m, p} ; m=0,1, \ldots, M-1\right\}$, where the time-domain samples $\bar{a}_{m, p^{\prime}}, m=0,1, \ldots, M-1$, are the latest average time-domain estimates for the $p^{\prime}$ th user transmitted symbols, i.e., the soft-decisions associated with the block of timedomain samples $\left\{\tilde{a}_{m, p^{\prime}} ; m=0,1, \ldots, M-1\right\}$, the IDFT of $\left\{\tilde{A}_{k, p^{\prime}} ; k=0,1, \ldots, M-1\right\}$. For the $i$ th iteration, $\bar{a}_{m, p^{\prime}}$ is associated with the $i$ th iteration for $p^{\prime}<p$ and with the $(i-1)$ th iteration for $p^{\prime} \geq p$ (in the first iteration, we do not have any information for $p^{\prime} \geq p$ and $\bar{a}_{m, p^{\prime}}=0$ ).

By extending the analytical approach of [7], it can be shown that the optimum feedforward coefficients in the MMSE sense can be written as

$$
\mathbf{F}_{p}(k)=\left[\mathbf{H}^{H}(k)\left(\mathbf{I}_{P}-\mathbf{P}^{2}\right) \mathbf{H}(k)+\alpha \mathbf{I}_{N}\right]^{-1} \mathbf{H}_{p}^{H}(k)
$$

$\left[(\cdot)^{H}\right.$ and $\mathbf{I}_{X}$ denote the Hermitian and size- $X$ identity matrices, respectively], with

$$
\alpha=\frac{E\left[\left|N_{k}\right|^{2}\right]}{E\left[\left|S_{k, p}\right|^{2}\right]},
$$

common to all $p$, and $\mathbf{P}=\operatorname{diag}\left(\rho_{1}, \cdots, \rho_{P}\right)(\operatorname{diag}(\cdot)$ denote the diagonal matrix), where the correlation coefficient $\rho_{p}$ is defined as [8]

$\rho_{p}=\frac{1}{M} \sum_{m=0}^{M-1} \frac{E\left[a_{m, p}^{*} \hat{a}_{m, p}\right]}{E\left[\left|a_{m, p}\right|^{2}\right]}=\frac{1}{2 M} \sum_{m=0}^{M-1}\left(\rho_{m, p}^{I}+\rho_{m, p}^{Q}\right)$,

with

$$
\rho_{m, p}^{I}=\frac{E\left[a_{m, p}^{I} \hat{a}_{m, p}^{I}\right]}{E\left[\left|a_{m, p}^{I}\right|^{2}\right]}=\tanh \left(\frac{\left|L_{m, p}^{I}\right|}{2}\right)
$$

and

$$
\rho_{m, p}^{Q}=\frac{E\left[a_{m, p}^{Q} \hat{a}_{m, p}^{Q}\right]}{E\left[\left|a_{m, p}^{Q}\right|^{2}\right]}=\tanh \left(\frac{\left|L_{m, p}^{Q}\right|}{2}\right),
$$

where $L_{m, p}^{I}$ and $L_{m, p}^{Q}$ denote the LLRs (LogLikelihood Ratios) of the "in-phase bit" and the "quadrature bit", associated to $a_{m, p}^{I}$ and $a_{m, p}^{Q}$, respectively, given $b^{2}$

$$
L_{m, p}^{I}=\frac{2}{\sigma_{p}^{2}} \tilde{a}_{m, p}^{I} \text { and } L_{m, p}^{Q}=\frac{2}{\sigma_{p}^{2}} \tilde{a}_{m, p}^{Q},
$$

respectively, where

$$
\sigma_{p}^{2}=\frac{1}{2} E\left[\left|a_{m, p}-\tilde{a}_{m, p}\right|^{2}\right] \approx \frac{1}{2 M} \sum_{m=0}^{M-1} E\left[\left|\hat{a}_{m, p}-\tilde{a}_{m, p}\right|^{2}\right] .
$$

$\rho_{m, p}^{I}$ and $\rho_{m, p}^{Q}$ can be regarded as the reliabilities associated to the "in-phase" and "quadrature" bits of the $m$ th symbol of the $p$ th user (naturally, $0 \leq \rho_{m, p}^{I} \leq 1$ and $0 \leq \rho_{m, p}^{Q} \leq 1$ ) (for the first iteration, $\rho_{m, p}^{I}=\rho_{m, p}^{Q}=0$ ).

The optimum feedback coefficients are given by

$$
\mathbf{B}_{p}(k)=\mathbf{P}\left(\mathbf{H}(k) \mathbf{F}_{p}(k)-\mathbf{I}_{P}\right) .
$$

If we do not have data estimates for the different users $\rho_{p^{\prime}}=0\left(p^{\prime}=1,2, \ldots, P\right)$ and the feedback coefficients are zero. Therefore, (16) reduces to

$$
\tilde{A}_{k, p}=\mathbf{F}_{p}^{T}(k) \mathbf{Y}(k),
$$

which corresponds to the linear receiver.

\section{Performance Results}

In this section, we present a set of performance results concerning the proposed iterative frequency-domain receiver that takes full advantage of the total bandwidth of the transmitted signals. We consider the uplink transmission within a CPassisted DS-CDMA scheme with a spreading factor $K=4$. Each of the $P$ users transmits $M$ data symbols corresponding to blocks of $N=K M=256$ samples within a total bandwidth $J$ times higher than the chip rate. The oversampled received signal corresponds to blocks of $J N$ samples. The duration of the useful part of the data blocks is $4 \mu \mathrm{s}$ and the $\mathrm{CP}$ has duration $1.1 \mu \mathrm{s}$. The data symbols are selected from a QPSK constellations under a Gray mapping rule. We consider a severely time-dispersive channel characterized by a

\footnotetext{
${ }^{2}$ It is assumed that the transmitted symbols are selected from a QPSK (Quaternary Phase Shift Keying) constellation under a Gray mapping rule (the generalization to other cases is straightforward).
} 
rectangular power delay profile with 64 rays separated by chip duration, with uncorrelated Rayleigh fading on the different paths. A linear power amplification is considered at each transmitter and perfect synchronization and channel estimation are assumed at the receiver. For the sake of comparisons, we included the MFB performance.

Let us first consider a scenario with $P=4,6$ or 8 users employing square-root raised-cosine pulses (i.e., their PSD has a raised-cosine shape). The roll-off factor is 1 , which means that the bandwidth is twice the chip rate (i.e., twice the minimum Nyquist bandwidth). Fig. 2 presents the average uncoded BER performance, averaged over all users, for iterations 1,2 and 4 (naturally, the first iteration corresponds to a linear FDE receiver). Clearly, the iterative receiver allows good interference suppression with significant performance improvement relatively to the conventional linear FDE after just 3 or 4 iterations. These gains are especially higher when the number of users and the corresponding interference levels are higher.

Fig. 3 shows the average uncoded BER performance, but corresponding to a scenario with $P=4,8$ or 12 users, employing a transmitter pulse shaping filter given by

$$
h^{(T)}(t)=\cos \left(\frac{\pi t}{T_{C}}\right) \operatorname{rect}\left(\frac{t}{T_{C}}\right) .
$$

In this case, the bandwidth of main lobe of the spectrum is three times larger than the symbol rate (i.e., three times the minimum Nyquist bandwidth), not to mention the residual side lobes. As in Fig. 2, it is possible to have twelve users sharing the band, although with some moderate performance degradation when we employ our iterative receiver.

Let us consider now a scenario where the power assigned to different users have different average powers at the receiver. We consider two classes of users, denoted by $C_{L}$ and $C_{H}$. The performance results presented in Fig. 4 concerns the case where we have a total of $P=8$ users, with 4 users in each

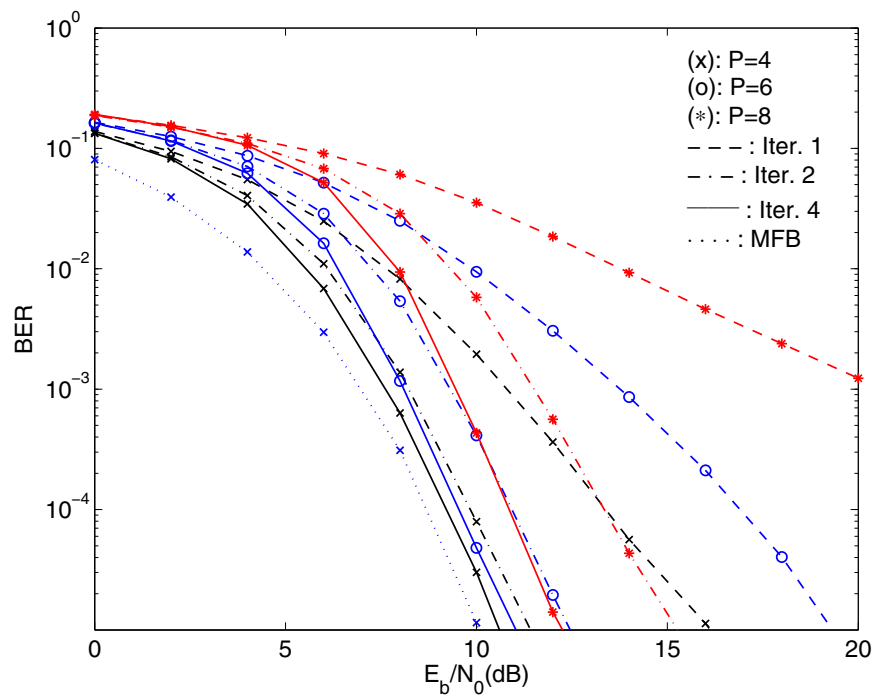

Fig. 2. Average uncoded BER performance for iterations 1,2 and 4 for $P=4,6$ or 8 users and square-root raised-cosine pulses with roll-off factor

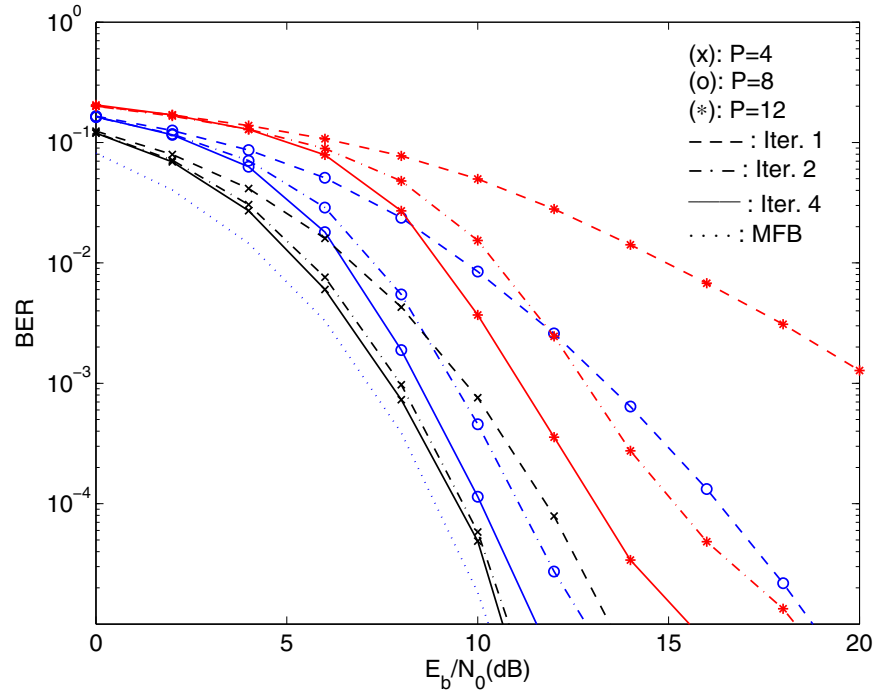

Fig. 3. As in Fig. 1, but for $P=4,8$ or 12 users with pulses given by (29).

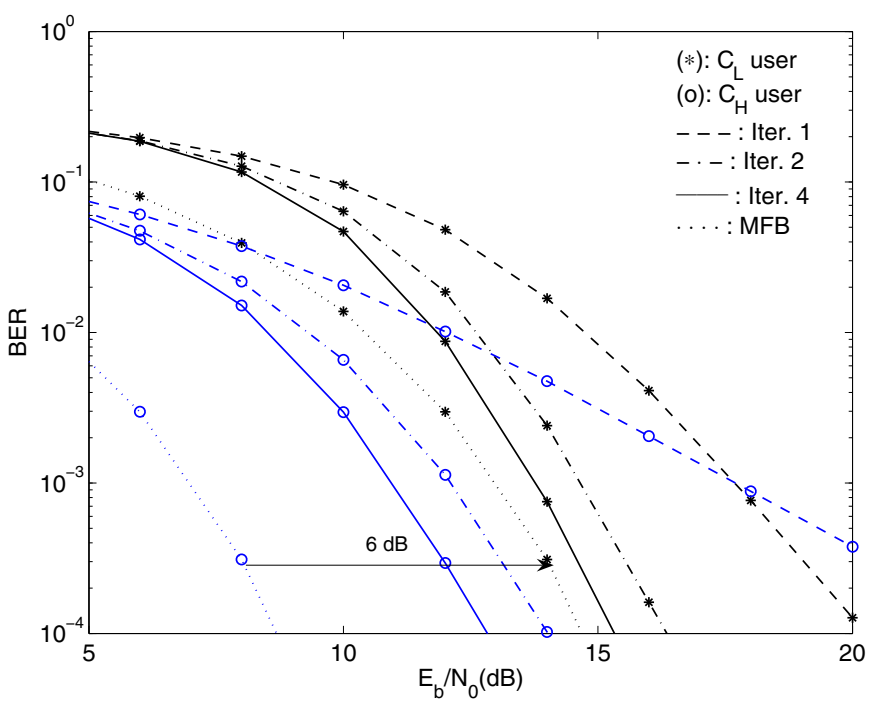

Fig. 4. Average BER performance (averaged over each class of users) for iterations 1,2 and 4 as a function of the $E_{b} / N_{0}$ of $C_{H}$ users (average power of $C_{H}$ users $6 \mathrm{~dB}$ above the average power of $C_{L}$ users), and square-root raised-cosine pulses with roll-off factor 1 .

class employing square-root raised-cosine pulses with rolloff factor 1. Fig. 5 concerns the case with $P=12$ users, with 6 users in each class employing pulses given by (29). In both cases, the average power of $C_{H}$ users is $6 \mathrm{~dB}$ above the average power of $C_{L}$ users. Clearly, the $C_{L}$ users face stronger interference levels. Once again, the proposed iterative receiver allows significant performance gain, specially for the low power users. The higher BER of low-power users preclude an appropriate interference cancelation when detecting highpower users.

\section{CONCLUSIONS}

In this paper we considered prefix-assisted DS-CDMA systems with bandwidth that can be significantly above the chip rate. To allow high spectral efficiency we considered highly overloaded systems where the number of users can be twice the spreading factor or even more. To cope with the strong 


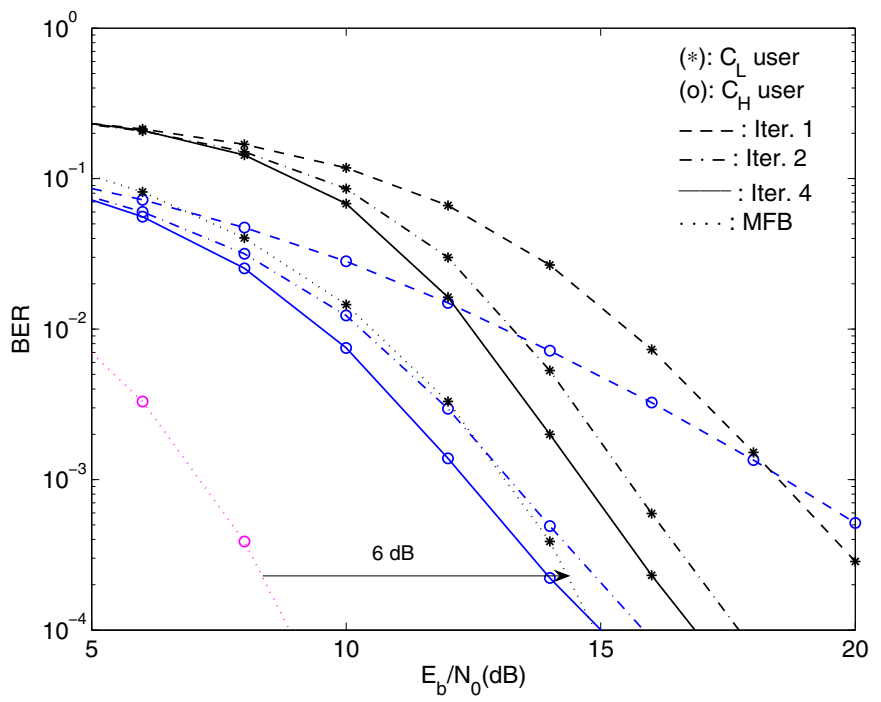

Fig. 5. Average BER performance (averaged over each class of users) for iterations 1,2 and 4 as a function of the $E_{b} / N_{0}$ of $C_{H}$ users (average power of $C_{H}$ users $6 \mathrm{~dB}$ above the average power of $C_{L}$ users), and pulses given by (29).

interference levels we presented an iterative frequency-domain receiver that takes full advantage of the total bandwidth of the transmitted signals. Our performance results show that the proposed receiver can have excellent performance, even for highly overloaded systems. Moreover, the overall system performance can be close to the maximum theoretical spectral efficiency, even with transmitted signals that have bandwidth significantly above the chip rate.

\section{REFERENCES}

[1] K. Baum, T. Thomas, F. Vook, V. Nangia, "Cyclic-Prefix CDMA: An Improved Transmission Method for Broadband DS-CDMA Cellular Systems", IEEE WCNC, pp. 183-188, 2002.

[2] A. Gusmão, R. Dinis, J. Conceição, and N. Esteves, "Comparison of Two Modulation Choices for Broadband Wireless Communications", IEEE VTC'OO (Spring), Tokyo, Japan, May 2000.

[3] D. Falconer, S. Ariyavisitakul, A. Benyamin-Seeyar and B. Eidson, "Frequency Domain Equalization for Single-Carrier Broadband Wireless Systems", IEEE Comm. Mag., Vol. 4, No. 4, pp. 58-66, April 2002.

[4] P. Silva and R. Dinis, "Frequency-Domain Multiuser Detection for CP-Assisted DS-CDMA Signals", In Proc. IEEE VTC'06 (Spring), Vol. 5, pp. 2103-2108, Melbourne, May 2006.

[5] N. Benvenuto, R. Dinis, D. Falconer and S. Tomasin, "Single Carrier Modulation with Non Linear Frequency Domain Equalization: An Idea Whose Time Has Come - Again", IEEE Proceedings, Vol. 98, No. 1, pp. 69-96, Jan. 2010.

[6] W. Gardner, "Exploitation of Spectral Redundancy in Cyclostationary Signals", IEEE Comm. Mag., Vol. 8, No. 2, pp. 14-36, Apr. 1991.

[7] R. Dinis, D. Falconer and B. Ng, "Iterative Frequency-Domain Equalizers for Adjacent Channel Interference Suppression", IEEE GLOBECOM'05, St. Louis, USA, Nov.-Dec. 2005.

[8] P. Silva and R. Dinis, "Turbo Detection for the Uplink of CPAssisted DS-CDMA Signals", IEEE PACRIM'07, Victoria, Aug. 2007. 